\title{
Graphene antidot lattices: Designed defects and spin qubits
}

Pedersen, Thomas; Flindt, Christian; Pedersen, Jesper Goor; Mortensen, Asger; Jauho, Antti-Pekka; Pedersen, Kjeld

Published in:

Physical Review Letters

Link to article, DOI:

10.1103/PhysRevLett.100.136804

Publication date:

2008

Document Version

Publisher's PDF, also known as Version of record

Link back to DTU Orbit

Citation (APA):

Pedersen, T., Flindt, C., Pedersen, J. G., Mortensen, A., Jauho, A-P., \& Pedersen, K. (2008). Graphene antidot lattices: Designed defects and spin qubits. Physical Review Letters, 100(13), 136804.

https://doi.org/10.1103/PhysRevLett.100.136804

\section{General rights}

Copyright and moral rights for the publications made accessible in the public portal are retained by the authors and/or other copyright owners and it is a condition of accessing publications that users recognise and abide by the legal requirements associated with these rights.

- Users may download and print one copy of any publication from the public portal for the purpose of private study or research.

- You may not further distribute the material or use it for any profit-making activity or commercial gain

- You may freely distribute the URL identifying the publication in the public portal

If you believe that this document breaches copyright please contact us providing details, and we will remove access to the work immediately and investigate your claim 


\title{
Graphene Antidot Lattices: Designed Defects and Spin Qubits
}

\author{
Thomas G. Pedersen, ${ }^{1}$ Christian Flindt, ${ }^{2,3}$ Jesper Pedersen, ${ }^{2}$ Niels Asger Mortensen, ${ }^{2}$ \\ Antti-Pekka Jauho, ${ }^{2,3}$ and Kjeld Pedersen ${ }^{1}$ \\ ${ }^{1}$ Department of Physics and Nanotechnology, Aalborg University, DK-9220 Aalborg East, Denmark \\ ${ }^{2}$ Department of Micro- and Nanotechnology, Technical University of Denmark, DTU Nanotech, \\ Building 345 East, DK-2800 Kongens Lyngby, Denmark \\ ${ }^{3}$ Laboratory of Physics, Helsinki University of Technology, P.O. Box 1100, FI-02015 HUT, Finland
}

(Received 2 November 2007; published 3 April 2008)

\begin{abstract}
Antidot lattices, defined on a two-dimensional electron gas at a semiconductor heterostructure, are a well-studied class of man-made structures with intriguing physical properties. We point out that a closely related system, graphene sheets with regularly spaced holes ("antidots"), should display similar phenomenology, but within a much more favorable energy scale, a consequence of the Dirac fermion nature of the states around the Fermi level. Further, by leaving out some of the holes one can create defect states, or pairs of coupled defect states, which can function as hosts for electron spin qubits. We present a detailed study of the energetics of periodic graphene antidot lattices, analyze the level structure of a single defect, calculate the exchange coupling between a pair of spin qubits, and identify possible avenues for further developments.
\end{abstract}

PACS numbers: 73.21.La, 03.67.Lx, 73.20.At

Graphene is the rapidly rising star of low-dimensional materials. Following the initial reports on fabrication by mechanical peeling [1] and epitaxial growth [2], this exceptional material has stimulated considerable experimental [3] and theoretical research [4] as well as proposals for novel electronic devices [5]. The promising prospects for graphene devices are based on several remarkable properties. Mainly, the sample quality and mobility (exceeding $15000 \mathrm{~cm}^{2} / \mathrm{V} \mathrm{s}$ [3]) can be very high. In addition, patterning of such monolayer films by $e$-beam lithography $[3,6]$ with features as small as $10 \mathrm{~nm}[3,7]$ is possible. Very recently, spintronics devices have been considered [8]. The incentive for graphene based spintronics lies partly in the long spin coherence time that is characteristic of carbonbased materials. This also has obvious advantages within the field of solid-state quantum information processing, where confined electron spins have been promoted as carriers of quantum information [9]. Being a light element, carbon has a rather small spin-orbit coupling, and, moreover, the predominant ${ }^{12} \mathrm{C}$ isotope has a vanishing hyperfine interaction. This makes graphene, at least in principle, a superior material compared to existing quantum computing implementations in GaAs [10,11].

Antidot lattices, defined on semiconductor heterostructures, display many intricate transport properties, in particular, in magnetic fields where the competing length scales lead to rich physics [12]. In this Letter we wish to draw attention to the possibility of forming antidot lattices on graphene. As mentioned above, state-of-the-art $e$-beam lithography has been used to carve graphene nanoribbons with feature sizes down to tens of nanometers. We propose to use similar techniques to create regular holes in the graphene sheet, in order to form antidot lattices. The antidot lattice has the important consequence that it turns the semimetallic graphene into a gapped semiconductor, where the size of the gap can be tuned via the antidot lattice parameters. As our analysis shall show, this electronic structure can be manipulated further so as to create coupled electron spin qubits, thus suggesting that these perforated graphene sheets are a promising platform for a large-scale spin qubit architecture. Localized spin qubit states can be formed in the antidot lattice by deliberately omitting some of the antidots. This idea has previously been analyzed for the two-dimensional electron gas in, e.g., GaAs heterostructures [13]. As we will now argue, moving to graphene has three major advantages: (i) increased coherence time; (ii) favorable energy scale of the defect states; and (iii) increased lateral confinement.

The proposed antidot lattice is simply a triangular array of holes in a graphene sheet, as illustrated in Fig. 1(a). The lattice consists of hexagonal unit cells as shown in Fig. 1(b), in which a roughly circular hole is created. We

a)

b)
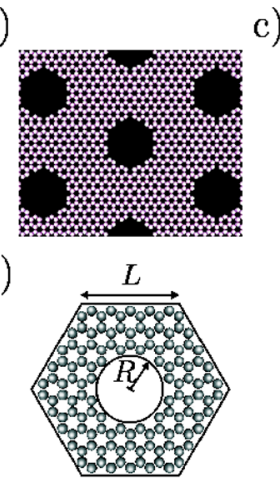

c)

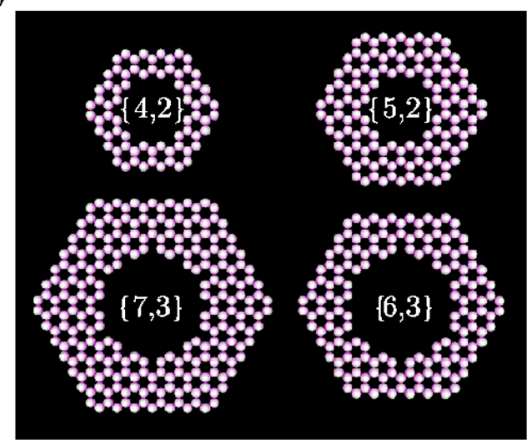

FIG. 1 (color online). Illustration of the triangular antidot lattice (a) with a unit cell characterized by side length $L$ and hole radius $R$ (b). In (c), several examples with corresponding $\{L, R\}$ parameters are shown. 
characterize the structure by the side length $L$ of the hexagonal unit cell and the radius $R$ of the hole, both measured in units of the graphene lattice constant $a \approx$ $2.46 \AA$. A lattice is designated by the notation $\{L, R\}$. Note that while $L$ is an integer, $R$ can be noninteger. As is evident from the examples in Fig. 1(c), $L$ is equal to the number of carbon atoms in the outermost row of the hexagon. Also of importance are the total number of sites in the unit cell $N_{\text {total }}$ (equal to the number of atoms before the hole is made) and the number of removed atoms $N_{\text {removed }}$. As an example, for the $\{7,3\}$ lattice $N_{\text {total }}=294$ and $N_{\text {removed }}=60$. Below, results for structures with $L \leq$ 14 and varying $R$ have been compiled taking care that no dangling bonds are formed, i.e., that all atoms have at least two neighbors. While these structures are too small for present-day lithography, results for realistic structures are easily obtained by simple scaling laws, as demonstrated below.

We model the structures using a tight-binding (TB) description considering a single $\pi$ orbital on each site and assuming a nearest-neighbor hopping integral of $-\beta$, with $\beta \approx 3.033 \mathrm{eV}$ [14]. In this description, energy levels are always distributed symmetrically above and below zero, which defines the Fermi level in the undoped case. The TB approximation is necessary due to the large antidot cells. It is known to accurately reproduce the low-energy part of the density-functional (DFT) band structure of graphene [15]. Edges, however, require a modification of hopping integrals near the edge to ensure agreement between DFT and TB calculations [16]. We have checked that the computed band structures are generally robust against such modifications, which simply produce a minor additional opening of the band gap. The electronic band structure and density of states for the $\{7,3\}$ structure are illustrated in Fig. 2. Importantly, a substantial energy gap of approximately $0.73 \mathrm{eV}$ opens around the Fermi level [17]. Hence, as hinted above, the periodic perturbation
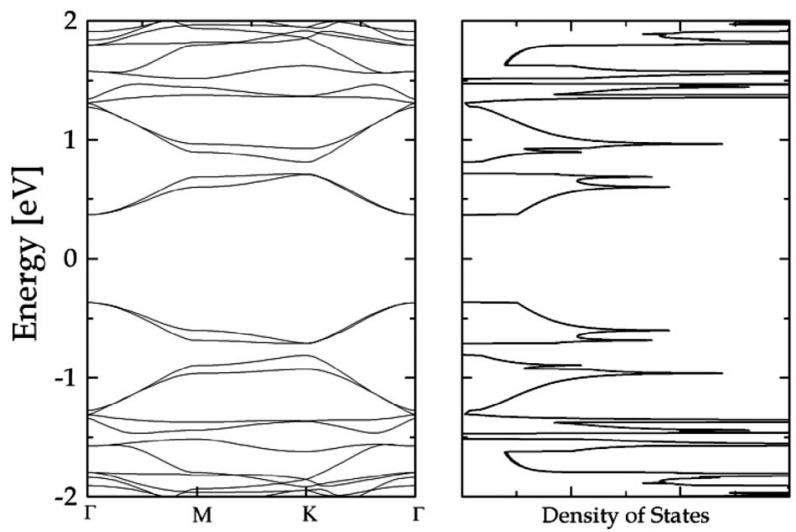

FIG. 2. Energy band structure and associated density of states for a $\{7,3\}$ antidot lattice. The notation $\Gamma, M$, and $K$ refers to high symmetry points of the Brillouin zone. turns the semimetal into a semiconductor. In the top panel of Fig. 3, band gaps $E_{g}$ of several structures are plotted versus the quantity $N_{\text {removed }}^{1 / 2} / N_{\text {total }}$. When plotted in this manner, a roughly linear behavior is observed. This simple result may be rationalized within the linearized Hamiltonian approximation treating electrons as massless Dirac fermions subject to the periodic perturbation of the antidot lattice. In this description, the wave function is a two-component spinor representing the two sublattices. The corresponding Hamiltonian is the $2 \times 2$ matrix operator

$$
H=\left(\begin{array}{cc}
V(x, y) & v_{F}\left(p_{x}-i p_{y}\right) \\
v_{F}\left(p_{x}+i p_{y}\right) & V(x, y)
\end{array}\right),
$$

where $V$ is the periodic antidot potential, $\mathbf{p}$ is the momentum operator, and the Fermi velocity $v_{F}=\sqrt{3} \beta a /(2 \hbar) \approx$ $10^{6} \mathrm{~m} / \mathrm{s}$. In the absence of a potential, the energy eigenvalues are simply $E= \pm \hbar v_{F}|\mathbf{k}|$. If the potential is approximated by infinite barriers at the positions of the antidots, the eigenvalue problem is reduced to the form

$$
v_{F}^{2}\left(p_{x}^{2}+p_{y}^{2}\right) \psi=E^{2} \psi,
$$

with the boundary condition that $\psi$ vanishes in the barrier region. The equation is mathematically similar to the usual effective mass equation. For an antidot lattice in a usual semiconductor material such as GaAs, simple scaling arguments lead to a band gap varying as $E_{g} \propto A_{\text {total }}^{-1} f\left(A_{\text {removed }} /\right.$ $A_{\text {total }}$, where $A_{\text {total }}$ is the area of the unit cell and $A_{\text {removed }}$ is the area removed inside each unit cell. In graphene, a similar behavior is expected except that the linear band structure changes the prefactor from $A_{\text {total }}^{-1}$ to $A_{\text {total }}^{-1 / 2}$, i.e.,

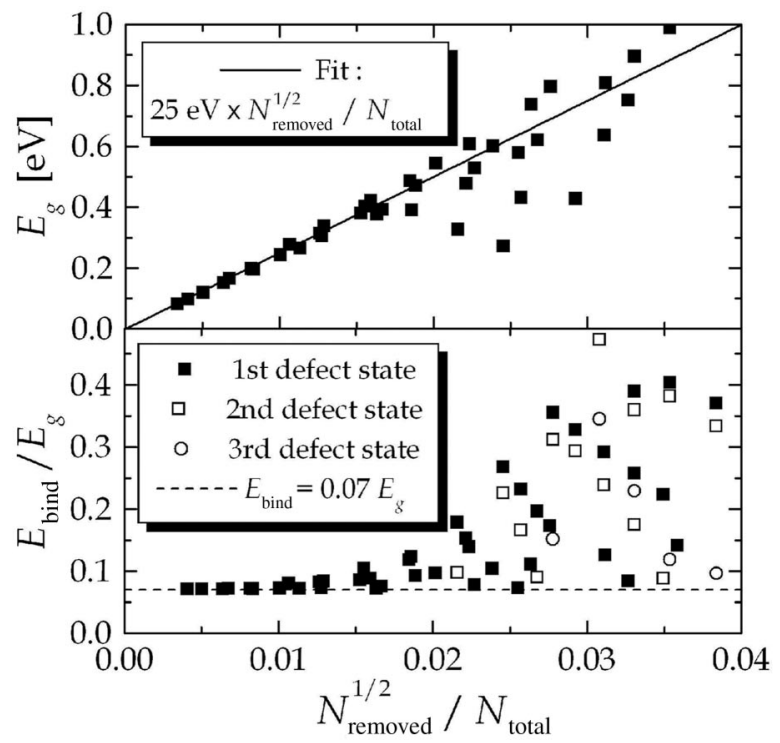

FIG. 3. Compilation of energy gaps (upper panel) and defect state binding energies (lower panel). When displayed versus $N_{\text {removed }}^{1 / 2} / N_{\text {total }}$, very simple scaling is observed. Note that $N_{\text {removed }}^{1 / 2} / N_{\text {total }}$ is small for realistic structures. 
$E_{g} \propto A_{\text {total }}^{-1 / 2} g\left(A_{\text {removed }} / A_{\text {total }}\right) \propto N_{\text {total }}^{-1 / 2} g\left(N_{\text {removed }} / N_{\text {total }}\right)$. The fit in Fig. 3 shows that $g$ approximately follows a square root behavior $g\left(N_{\text {removed }} / N_{\text {total }}\right) \propto \sqrt{N_{\text {removed }} / N_{\text {total }}}$. Thus, the net result is a gap varying as $E_{g} \approx K \times$ $N_{\text {removed }}^{1 / 2} / N_{\text {total }}$ with a constant $K \approx 25 \mathrm{eV}$. For large unit cells, $N_{\text {removed }}^{1 / 2} / N_{\text {total }}$ is small and in this case the linear fit is an excellent approximation. The weaker scaling $\left(A_{\text {total }}^{-1 / 2}\right.$ instead of $A_{\text {total }}^{-1}$ ) of graphene is very favorable for the purpose of obtaining large band gaps even for relatively large structures. The practical limits of present-day $e$-beam lithography probably restrict the obtainable size of the unit cell to around $10 \mathrm{~nm}$ across corresponding to a total number of carbon atoms of $N_{\text {total }} \approx 3000$. Assuming $N_{\text {removed }} \approx N_{\text {total }} / 4$ we find a substantial gap of $0.23 \mathrm{eV}$. Hence, band gaps much larger than the thermal energy at room temperature are certainly realistic. This feature, which is a direct consequence of the massless Dirac fermion behavior, is very important for the feasibility of the graphene based devices considered here.

We now turn to the role of intentional defects in the antidot lattice produced by leaving one or several unit cells intact, i.e., without a hole. Such defects may support localized electronic states and may consequently be utilized for electron spin qubits, as we will now demonstrate. An example of single and double defects for the $\{5,2\}$ structure is shown in Fig. 4. For isolated single defects, we compute localized states by periodically replicating the supercell consisting of one intact and six perforated cells illustrated in the figure. The states are sufficiently localized that cross talk between neighboring super cells is negligible. Periodicity is not crucial for the appearance of bound states [13]. Defect states are identified by an energy lying in the fundamental energy gap, i.e., the gap containing the

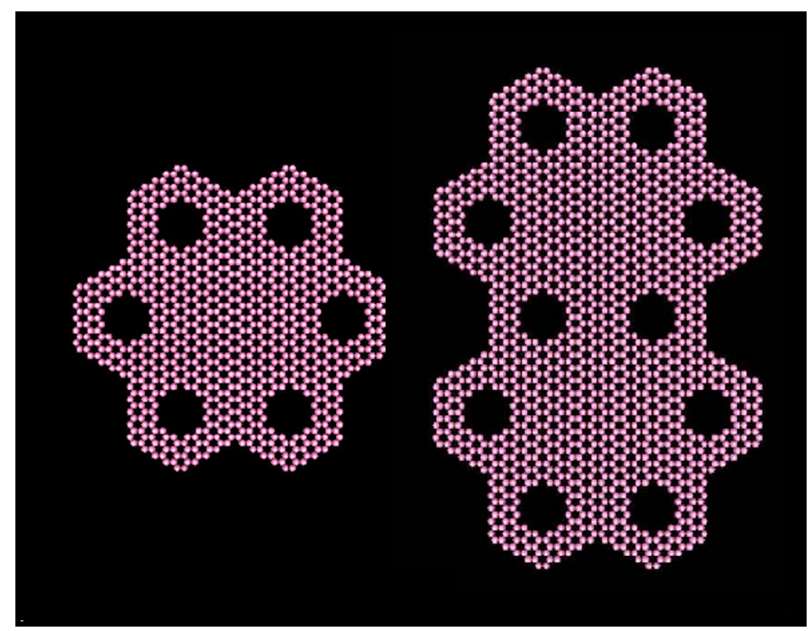

FIG. 4 (color online). Single (left) and double (right) defects for the $\{5,2\}$ antidot lattice. To compute defect states, super cells containing defects surrounded by six intact units are repeated periodically.
Fermi energy. In fact, other energy gaps may exist as illustrated in Fig. 2; here we focus solely on states in the fundamental gap. If the gap is sufficiently large (i.e., if $N_{\text {removed }}^{1 / 2} / N_{\text {total }}$ is large) several defect states are supported. In the lower panel of Fig. 3, a compilation of binding energies for the three lowest defect states is shown. We define the binding energy $E_{\text {bind }}$ as the downwards shift of the defect state energy measured from the conduction band edge. Hence, a defect state at the Fermi energy would have a binding energy of $E_{g} / 2$. For small band gaps, only a single-defect state is supported but several defect states appear in an irregular pattern as the confinement increases. Note that the scatter in the data points in the plot reflects actual variations and not computational inaccuracy. Importantly, the binding energy in the limit of small band gaps is seen to approach a constant fraction $\simeq 0.07$ of the energy gap. Hence, for the $10 \mathrm{~nm}$ unit cell considered above, a defect state would be bound by roughly $16 \mathrm{meV}$. This implies that liquid nitrogen cooling should be sufficient to observe these states.

Next, we consider two tunnel coupled defect states in a "double defect," illustrated in Fig. 4. With an electron occupying a nondegenerate state in each defect, the spins of the two electrons couple due to the exchange interaction $J \mathbf{S}_{1} \cdot \mathbf{S}_{2}$. If the two single-defect states are energetically aligned, the exchange coupling is given as $J=4 t^{2} / U$ according to the Hubbard approximation. Here, $t$ is the tunnel coupling between the two defect states, and $U$ is the single-defect Coulomb integral. As discussed in Ref. [9], the exchange coupling constitutes a key element in quantum computing architectures based on electron spins as qubits, enabling interactions between different qubits. Importantly, the exchange coupling can be controlled with external gate potentials. Metallic gates could be realized by lithographic methods and placed either below or on top of the graphene sheet but will not be considered further here. For evaluation of the exchange coupling, we calculate the single-defect Coulomb integral $U$ by the method presented in Ref. [18] (ignoring overlap between different atomic $\pi$ orbitals) using the Ohno form to interpolate between the intra- and long-range interatomic Coulomb coupling. A Hubbard $U_{\pi}$ for carbon $\pi$ orbitals of $20.08 \mathrm{eV}$ [18] and dielectric constant of 2.5 [19] (as appropriate for graphene on $\mathrm{SiO}_{2}$ ) are applied. The tunnel coupling $t$ is extracted from the single-particle energy spectrum.

Our findings for the Coulomb integral $U$ are illustrated in Fig. 5. In the plot, $R_{D}$ is the effective defect radius calculated by including half the area of the surrounding cells and writing the total area as $\pi R_{D}^{2}$. The smallest $U$ 's are found for the least localized states for which $U$ scales as the expected $R_{D}^{-1}$. The inset shows, as an example, the single-electron level diagram for single and double defects in a $\{12,7\}$ lattice. This structure has $N_{\text {total }}=864$ and $N_{\text {removed }}=348$ and supports two single-defect states. Of these, the upper one is nondegenerate and the Coulomb 


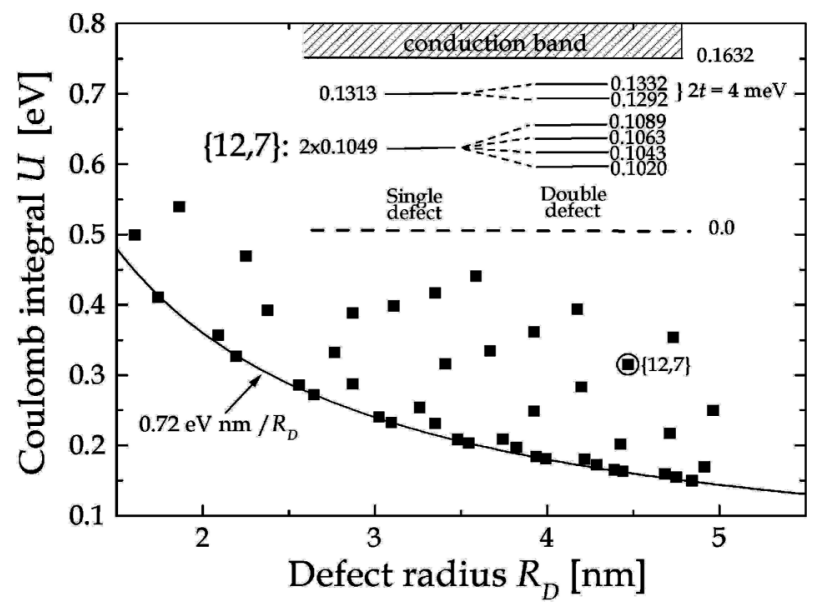

FIG. 5. Compilation of Coulomb integrals $U$ for single-defect states showing roughly linear scaling with $R_{D}^{-1}$ for delocalized states. Inset: Level structure for single and double defects in a $\{12,7\}$ lattice. The Coulomb integral for this case is indicated by the circle.

integral is $0.315 \mathrm{eV}$. Because of the large double defect super cell, this is about the largest structure that we have been able to analyze. As shown, the level splitting corresponds to a tunnel coupling of $t \approx 2 \mathrm{meV}$ between the two nondegenerate single-defect states. Hence, based on the $\{12,7\}$ values we may estimate the exchange coupling to be on the order of $J \approx 50 \mu \mathrm{eV}$. Naturally, this value could be tuned by appropriate design of the barrier region that, for simplicity, has been constructed from two intact unit cells. Also, going to larger single defects would decrease $U$ and, in turn, increase $J$. Note, however, that $t$ depends exponentially on barrier width whereas $U$ is only weakly dependent on geometry. Hence, the geometric influence on $J$ will be determined mainly through $t$ rather than $U$.

We believe that the approach outlined above can be extended to more complicated structures. Going from a single pair of spin qubits in an isolated double defect to several coupled spins could be achieved with little added complication. Similarly, a double defect could be replaced by a linear array of defects. Hence, the number of qubits can be increased essentially without complicating the fabrication procedure. In practice, excellent control of the $e$-beam lithography process remains a critical issue.

In summary, we have shown that antidot lattices pave the way for controlled manipulation of the electronic properties of graphene sheets. The material can be rendered semiconducting with a significant and controllable energy gap. The magnitude of the gap is explained by a simple scaling argument and could reach several tenths of $\mathrm{eVs}$ for realistic structures. Introducing defects into the antidot lattice leads to the formation of localized electronic states. Combined with the extremely long spin coherence time of carbon-based materials this could lead to a practical realization of spin qubits. With a properly designed double defect, two-electron states derived from defect levels near the Fermi level are found to fulfil the requirements for such qubits.

A.P. J. and C. F. acknowledge the FiDiPro program of the Finnish Academy of Sciences for support during the final stages of this work. We thank Dr. A. Krasheninnikov for an informative discussion on gap states in graphene.

[1] K. S. Novoselov et al., Science 306, 666 (2004).

[2] C. Berger et al., J. Chem. Phys. B 108, 19912 (2004).

[3] A. K. Geim and K.S. Novoselov, Nat. Mater. 6, 183 (2007).

[4] M. I. Katsnelson, K. S. Novoselov, and A. K. Geim, Nature Phys. 2, 620 (2006); V. V. Cheianov, V. Fal'ko, and B. L. Altshuler, Science 315, 1252 (2007).

[5] A. Rycerz, J. Tworzydlo, and C. W. J. Beenakker, Nature Phys. 3, 172 (2007).

[6] C. Berger et al., Science 312, 1191 (2006).

[7] M. Y. Han, B. Özyilmaz, Y. Zhang, and P. Kim, Phys. Rev. Lett. 98, 206805 (2007).

[8] E. W. Hill et al., IEEE Trans. Magn. 42, 2694 (2006); Y. W. Son, M. L. Cohen, and S. G. Louie, Nature (London) 444, 347 (2006); T. B. Martins, R. H. Miwa, A. J. R. da Silva, and A. Fazzio, Phys. Rev. Lett. 98, 196803 (2007).

[9] D. Loss and D. P. DiVincenzo, Phys. Rev. A 57, 120 (1998); G. Burkard, D. Loss, and D. P. DiVincenzo, Phys. Rev. B 59, 2070 (1999); D. P. DiVincenzo et al., Nature (London) 408, 339 (2000).

[10] B. Trauzettel, D. V. Bulaev, D. Loss, and G. Burkard, Nature Phys. 3, 192 (2007).

[11] J. R. Petta et al., Science 309, 2180 (2005).

[12] D. Weiss et al., Phys. Rev. Lett. 66, 2790 (1991).

[13] C. Flindt, N. A. Mortensen, and A. P. Jauho, Nano Lett. 5, 2515 (2005); J. Pedersen, C. Flindt, N. A. Mortensen, and A. P. Jauho, Phys. Rev. B 77, 045325 (2008).

[14] R. Saito, G. Dresselhaus, and M. S. Dresselhaus, Physical Properties of Carbon Nanotubes (Imperial College Press, London, 1998).

[15] S. Reich, J. Maultzsch, C. Thomsen, and P. Ordejón, Phys. Rev. B 66, 035412 (2002).

[16] Y. W. Son, M. L. Cohen, and S. G. Louie, Phys. Rev. Lett. 97, 216803 (2006).

[17] This prediction is unchanged, if one modifies the tightbinding parameters along the edges of the perforation, as suggested in Ref. [16]. Also, perfect periodicty of the holes is not crucial as discussed in Ref. [13].

[18] T. G. Pedersen, Phys. Rev. B 69, 075207 (2004).

[19] T. Ando, J. Phys. Soc. Jpn. 75, 074716 (2006). 